\title{
Natural Compounds for the Management of Parkinson's Disease and Attention-Deficit/Hyperactivity Disorder
}

\author{
Juan Carlos Corona \\ Laboratory of Neurosciences, Hospital Infantil de México Federico Gómez, Mexico \\ Correspondence should be addressed to Juan Carlos Corona; jcorona@himfg.edu.mx
}

Received 28 June 2018; Revised 31 October 2018; Accepted 11 November 2018; Published 22 November 2018

Guest Editor: Francesco Facchiano

Copyright (C) 2018 Juan Carlos Corona. This is an open access article distributed under the Creative Commons Attribution License, which permits unrestricted use, distribution, and reproduction in any medium, provided the original work is properly cited.

\begin{abstract}
Parkinson's disease (PD) is the second most common neurodegenerative disorder with an unknown aetiology. The pathogenic mechanisms include oxidative stress, mitochondrial dysfunction, protein dysfunction, inflammation, autophagy, apoptosis, and abnormal deposition of $\alpha$-synuclein. Currently, the existing pharmacological treatments for PD cannot improve fundamentally the degenerative process of dopaminergic neurons and have numerous side effects. On the other hand, attention-deficit/hyperactivity disorder (ADHD) is the most common neurodevelopmental disorder of childhood and is characterised by hyperactivity, impulsivity, and inattention. The aetiology of ADHD remains unknown, although it has been suggested that its pathophysiology involves abnormalities in several brain regions, disturbances of the catecholaminergic pathway, and oxidative stress. Psychostimulants and nonpsychostimulants are the drugs prescribed for the treatment of ADHD; however, they have been associated with increased risk of substance use and have several side effects. Today, there are very few tools available to prevent or to counteract the progression of such neurological disorders. Thus, therapeutic approaches with high efficiency and fewer side effects are needed. This review presents a brief overview of the two neurological disorders and their current treatments, followed by a discussion of the natural compounds which have been studied as therapeutic agents and the mechanisms underlying the beneficial effects, in particular, the decrease in oxidative stress.
\end{abstract}

\section{Introduction}

For many years, natural compounds have provided an efficient resource for the discovery of potential therapeutic agents. Among them, many natural products possess antioxidative, antiapoptotic and anti-inflammatory activities. In this review, we summarize the role of natural compounds and their therapeutic use for the management of dopaminerelated diseases, divided into part 1 for Parkinson's disease and part 2 for attention-deficit/hyperactivity disorder. In addition, the current knowledge of the mechanisms underlying the potential beneficial effects of diverse natural compounds capable of counteracting the progression of this type of diseases is reviewed, as demonstrated by their antioxidative effects.

\section{Parkinson's Disease}

Parkinson's disease (PD) is the second most common progressive and chronic neurodegenerative disorder, characterised by the progressive loss of dopaminergic neurons in the substantia nigra (SN) and their projections to the striatum. Thus, the function of the nigrostriatal pathway becomes reduced and causes the development of movement disorder [1]. The basic characteristics of PD include tremor, rigidity, bradykinesia, and impaired balance; depression is also present in patients, affecting the quality of life. One of the pathological features of PD is the presence of Lewy bodies, which are intraneuronal proteinaceous cytoplasmic inclusions and include $\alpha$-synuclein, ubiquitin, and neurofilaments found in all affected brain regions [2]. The pathogenic mechanisms of PD include oxidative stress, mitochondrial dysfunction, protein dysfunction, inflammation, autophagy, and apoptosis [3]. PD occurs $95 \%$ as a sporadic form, while familial forms involve mutations in proteins that include PINK1, DJ-1, PARKIN, FBXO7, and LRRK2 [4], even though pesticides, chemicals, and metals may increase the risk of developing PD. Currently, the treatment of PD includes drugs such as L-DOPA, which is catalysed primarily by dopa decarboxylase in the brain and is 
converted into dopamine, producing its therapeutic effects. Another treatment includes anticholinergic drugs that can block striatal cholinergic receptors inhibiting the excitability of cholinergic nerves; it has also been demonstrated that they can inhibit dopamine reuptake to enhance the function of dopaminergic neurons [5]. At present, there are also other drugs in Phase III clinical trials. Nevertheless, the current drugs used for the PD treatment have some side effects, limiting their clinical applications [6]. Drugs used for the PD treatment and their side effects are shown in Table 1. Thus, the growing interest in alternative therapies for neurodegenerative disorders, including PD, has focused on the neuroprotective and antioxidant effects of natural products that may provide alternatives, since they can have high efficiency and fewer side effects. Natural compounds used as alternative therapies for the management of PD are shown in Table 2.

2.1. Ginkgo biloba. Ginkgo biloba is an ancient tree native to China and has been extensively used in traditional Chinese medicine to manage symptoms associated with dysfunctions of the heart and lungs. G. biloba usually contains three ingredients, which include flavonoids, terpenoids, and ginkgolic acid [81]. Ginkgolides well-known plant extracts obtained from leaves from G. biloba, especially in the preparation EGb761, which contains ginkgolide $\mathrm{B}$ and bilobalide, have emerged as natural therapeutic compounds, in part due to their antioxidant activity. These effects have been observed in the 1-methyl-4-phenyl-1,2,3,6-tetrahydropyridine- (MPTP-) treated mouse model of PD, where chronic ingestion of EGb761 prevented MPTP-induced reduction in the dopaminergic nerve endings [9]. In addition, EGb761 administered before or after MPTP treatment protected against MPTPinduced dopaminergic neurotoxicity [10]. Moreover, EGb761 attenuated the neurotoxic effect of levodopa in the 6hydroxydopamine (6-OHDA) model of PD, indicated that levodopa is neurotoxic and that EGb761 may decrease this toxicity [11]. The neuroprotective effects of EGb761 were demonstrated in the 6-OHDA rat model, as indicated by the reduction in the behavioural deficit in the rat [12]. Paraquat is a pesticide that has been linked to $\mathrm{PD}$, and it has been demonstrated that EGb761 protects against paraquat-induced apoptosis of PC12 cells by increasing bcl-2 activation, maintaining of mitochondrial membrane potential $\left(\Delta \Psi_{\mathrm{m}}\right)$ and decreasing caspase-3 activation through the mitochondria-dependent pathway [13]. The neuroprotective effect of EGb761 against MPTP neurotoxicity is associated with the blockade of lipid peroxidation, reduction of oxidative stress, and attenuation of MPTP-induced neurodegeneration of the nigrostriatal pathway [14]. Also, it was demonstrated in an extensive review that EGb761 may exert therapeutic actions in an animal model of PD via the antioxidant effects [82]. Ginkgetin, a natural biflavonoid isolated from leaves of G. biloba, protected against 1-methyl-4-phenylpyridinium ion- $\left(\mathrm{MPP}^{+}-\right)$induced cell damage in vitro by decreasing the levels of intracellular reactive oxygen species (ROS) and by maintaining $\Delta \Psi_{\mathrm{m}}$ and also improved the sensorimotor coordination in a mouse PD model induced by MPTP, suggesting that the neuroprotective mechanism of ginkgetin occurs via regulating iron homeostasis [16]. In low-dose whole-body $\gamma$-irradiation in the reserpine model of PD, EGb761 was protective by ameliorating the reserpine-induced state of oxidative stress, mitochondrial dysfunction, and apoptosis in the brain [15]. The pretreatment with ginkgolide $\mathrm{B}$ or bilobalide protected SH-SY5Y cells against $\alpha$-synuclein-induced cell injury and apoptosis [17]. Ultimately, the G. biloba extract treatment improved locomotor activity, decreased oxidative damage, maintained the dopamine homeostasis, and inhibited the development of PD in A53T $\alpha$-synuclein transgenic mice [18].

2.2. Ginseng. Ginseng is a traditional Chinese herb containing more than 30 ginsenosides, the active ingredients of ginseng. Ginsenosides Rb1 and Rgl are regarded as the main compounds responsible for the therapeutic actions of ginseng. Previous studies have shown that, in SN-K-SH cells, both ginsenosides Rb1 and Rg1 reversed MPTP-induced cell death [19]. The protective effect of Rgl against MPTPinduced apoptosis was attributed to enhancing Bcl-2 and $\mathrm{Bcl}-\mathrm{xl}$ expression, reducing Bax and iNOS expression, and inhibiting activation of caspase-3 [20]. The ginseng extract G115 significantly blocked tyrosine hydroxylase- (TH-) positive cell loss in the $\mathrm{SN}$ and reduced the appearance of locomotor dysfunction in two rodent models of PD [21]. Pretreatments of Rg1 or $\mathrm{N}$-acetylcysteine were found to protect against MPTP-induced SN neuron loss by preventing glutathione (GSH) reduction, attenuate the phosphorylations of JNK and c-Jun, and activate superoxide dismutase (SOD) [22]. $\mathrm{MPP}^{+}$-induced cytotoxicity in SH-SY5Y was inhibited by water extract of ginseng, as demonstrated by the inhibitory effect on cell death, overproduction of ROS, elevated Bax/Bcl2 ratio, release of cytochrome $c$, and activation of caspase3 expression [23]. It was demonstrated that ginsenosides protect by reducing intracellular ROS levels, enhancing antioxidant activity, preserving the activity of complex I, stabilising the $\Delta \Psi_{\mathrm{m}}$, and increasing intracellular ATP levels. Rg1 treatment restored motor functions in MPTP-treated mice and these behavioural ameliorations were accompanied by restoration of dopaminergic neurons in the SN and striatum [24]. Additionally, the ginsenoside Rbl exhibited a strong ability to disaggregate fibrils and to inhibit the polymerisation of $\alpha$-synuclein [25]. Rg1 treatment inhibited the activation of microglia and reduced the infiltration of CD3+ and T cells and also protected TH-positive cells in the $\mathrm{SN}$ and reduced the serum concentrations of proinflammatory cytokines TNF $\alpha$, IFN $\gamma$, IL-1 $\beta$, and IL-6 in MPTP mouse models [26]. Also, using in vivo and in vitro models of $\mathrm{PD}$, it was demonstrated that ginsenoside Rg1 may exert therapeutic effects on $\mathrm{PD}$ via the $\mathrm{Wnt} / \beta$-catenin signalling pathway [27]. In the MPTP-probenecid mouse model, the oral treatment with Rg1 significantly attenuated MPTP-induced mortality, behaviour defects, and loss of dopaminergic neurons. The protective effect of Rg1 may be mediated by reducing aberrant $\alpha$ synuclein-mediated neuroinflammation [28]. The neurotoxin rotenone is an inhibitor of complex I and has been widely used in vivo and in vitro to model PD. Thus, cotreatment with ginsenosides $\mathrm{Rd}$ and $\mathrm{Re}$ inhibited the increased intracellular ROS production and lipid peroxidation accumulation caused by rotenone. Besides, the major ginsenosides $\mathrm{Rd}$ and $\mathrm{Re}$ 


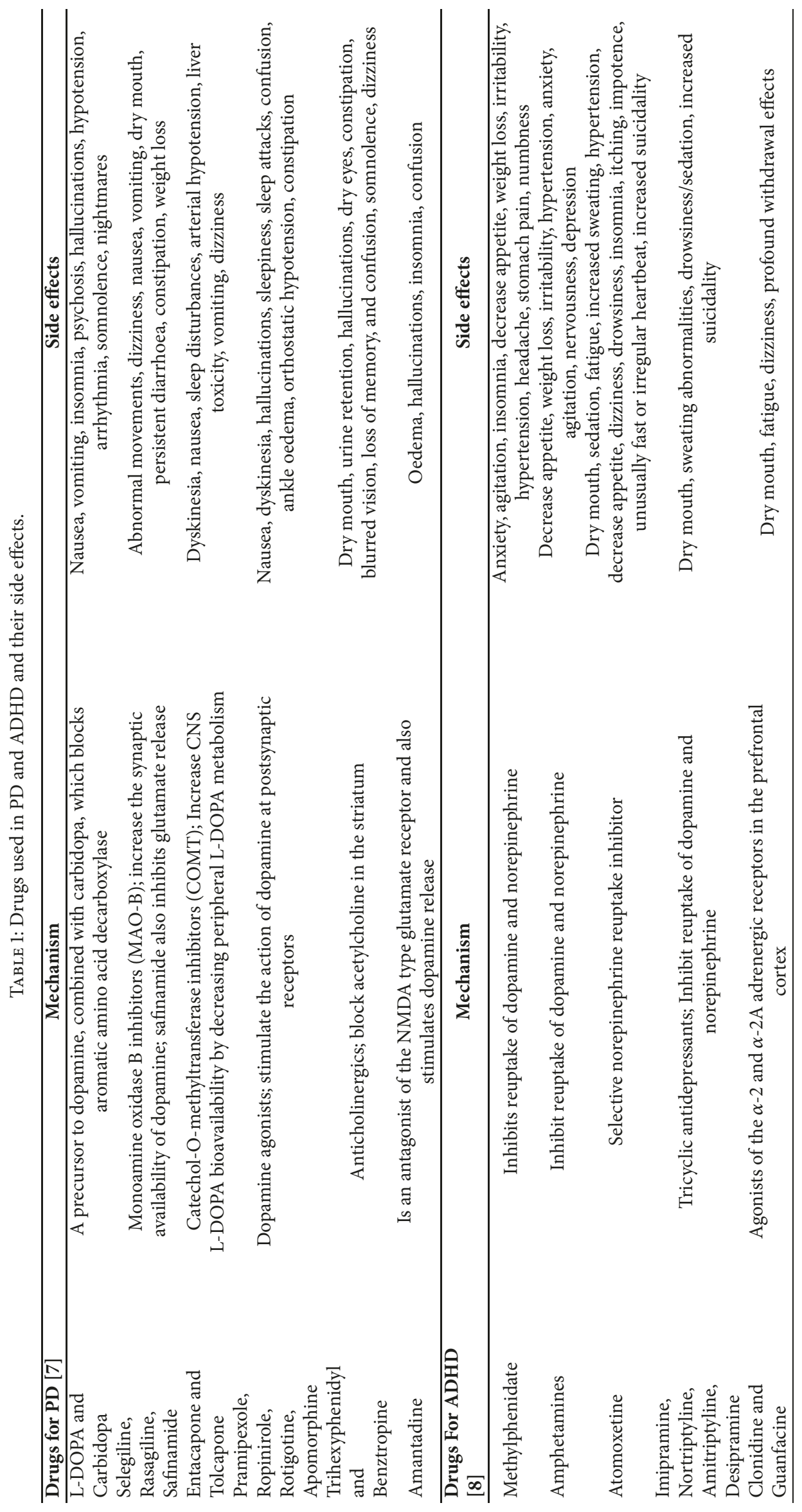




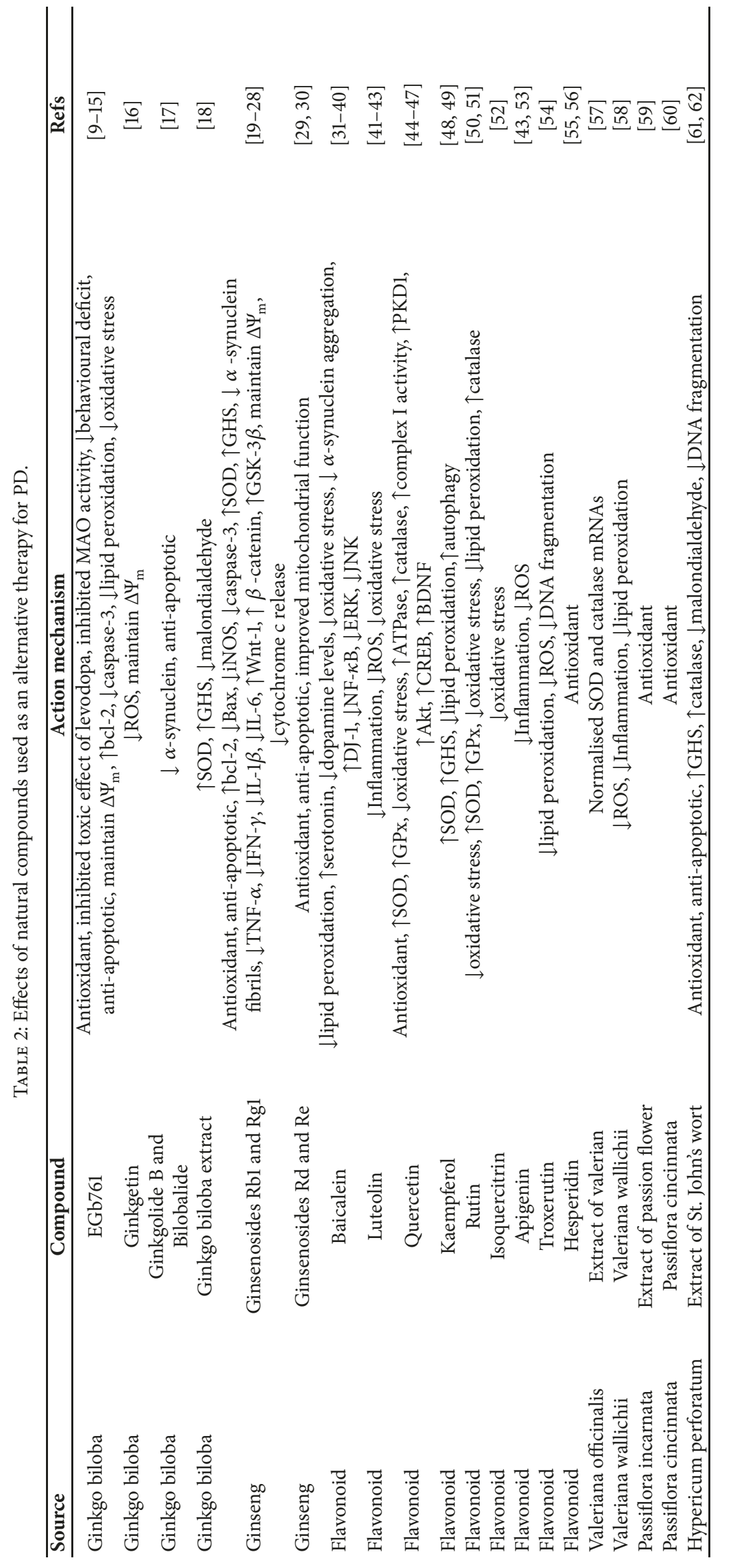


upregulate SOD and aconitase activities, and GSH also attenuates the depolarisation of $\Delta \Psi_{\mathrm{m}}$ and restores $\mathrm{Ca}^{2+}$ levels and moreover prevents apoptosis by modulating Bax and $\mathrm{Bcl}-2$ and inhibiting cytochrome $\mathrm{c}$ release and caspase3 activation [29]. Additionally, ginsenoside Rd reversed the loss of TH-positive cells in SN in vivo and in vitro $\mathrm{PD}$ models, which may involve its antioxidant effects and mitochondrial function preservation [30].

2.3. Flavonoids. Flavonoids are part of a large group of natural polyphenol phytochemicals with a long history of use as therapeutic agents. Baicalin, a flavonoid isolated from Scutellaria baicalensis, is the main metabolite of baicalein. The neuroprotective efficacy of baicalein has been shown in an in vivo model of PD using the neurotoxin 6-OHDA, where the protective effects on dopaminergic dysfunction and lipid peroxidation were seen [31]. Other reports showed that baicalein prevented abnormal behaviour by increasing dopaminergic neurons and dopamine and serotonin levels in the striatum and also inhibited oxidative stress and astroglial response [32]. Luteolin and apigenin are flavones with similar structure; luteolin is found in celery, broccoli, parsley, thyme, and olive oil, and apigenin is present in vegetables, several fruits, and herbs. Luteolin protects dopaminergic neurons against inflammation-induced neurotoxicity by inhibiting microglial activation [41]. Moreover, baicalein exerts protective effects in vivo and in vitro against 6-OHDA [33]. In addition, baicalein protects cells against the toxicity of a point mutation in $\alpha$ synuclein [34]. Baicalein also inhibited the formation of $\alpha$ synuclein oligomers and consequently prevents its oligomerisation [35]. Quercetin is the aglycone form of a number of other flavonoid glycosides, such as rutin and quercitrin, and is found in citrus fruit, onions, and grains. In the 6OHDA rat model, treatment of quercetin increased levels of antioxidant and striatal dopamine and reduced dopaminergic neuronal loss [44]. Kaempferol is a natural flavonoid which has been found in grapefruit, and other plant sources. It improves motor coordination, raises striatal dopamine and its metabolite levels, increases SOD and GSH activity, and reduces the content of lipid peroxidation, also preventing the loss of TH-positive neurons induced by MPTP [48]. There is also evidence of neuroprotection by kaempferol by autophagy in SH-SY5Y cells and primary neurons against rotenone toxicity [49]. It has been demonstrated that rutin protects dopaminergic neurons against oxidative stress induced by 6-OHDA [50]. Furthermore, it has been shown that quercetin protects against oxidative stress and increases activities of glutathione peroxidase (GPx), SOD, ATPase, AchE, and dopamine depletion in MPTP-treated mice [45]. The bioflavonoid rutin inhibits 6-OHDA-induced neurotoxicity in PC12 cells by activation of SOD, catalase, GPx, and total GSH and by inhibition of lipid peroxidation [51]. Moreover, in a rotenone model, quercetin has been shown to upregulate mitochondrial complex I activity and increase catalase and SOD activity [46]. Mitochondrial dysfunction in SH-SY5Y cells and upregulation of DJ-1 protein expression induced by 6-OHDA is prevented by baicalein [36]. The flavonol isoquercitrin protects $\mathrm{PC} 12$ cells against 6-OHDA-induced oxidative stress [52]. Baicalein downregulates the activation of NF- $\kappa \mathrm{B}, \mathrm{ERK}$, and JNK and attenuates astrocyte activation in MPTP mice [37]. Baicalein inhibits the upregulation of proinflammatory cytokines in the SN and striatum in PD mice models [38]. Luteolin also reduces cytotoxicity induced by 6-OHDA and ROS production in neuronal PC12 cells by modulating changes in the stress response pathway [42]. In MPTP-treated mice, luteolin and apigenin protect dopaminergic neurons by reducing oxidative damage, neuroinflammation, and microglial activation and also improve muscular and locomotor activity [43]. The flavonoids and their metabolites can interact with neuronal receptors and modulate kinase signalling pathways, transcription factors, and gene and/or protein expression, which control memory and learning processes in the hippocampus [83]. Naringin is a flavonoid glycoside that is contained abundantly in the skin of grapefruit and orange and is the origin of their bitterness. It protects dopaminergic neurons by induction of the activation of mammalian target of rapamycin complex 1 (mTORCl) and inhibited microglial activation in the SN of the mouse treated with 6-OHDA [84]. In a rotenone mouse model, baicalein prevented the progression of $\alpha$-synuclein accumulation and protected dopaminergic neurons and also inhibited the formation of $\alpha$-synuclein oligomers [39]. It has been shown that baicalein inhibits $\alpha$-synuclein aggregates and autophagy in rats treated with $\mathrm{MPP}^{+}[40]$. Besides, in the same model, apigenin ameliorated dopaminergic neuronal loss and improved behavioural, biochemical, and mitochondrial enzyme activities; such effects were associated with the suppression of oxidative stress and neuroinflammation [53]. Recently, it was demonstrated that quercetin improved mitochondrial biogenesis and induced the activation of two major cell survival kinases, PKD1, and Akt and also enhanced CREB and BDNF (a CREB target gene) in MN9D cells against 6-OHDA-induced neurotoxicity, and in the MitoPark transgenic mouse model of PD, quercetin improved behavioural deficits and reduced TH cell loss [47]. Troxerutin (also known as vitamin $\mathrm{P} 4$ ) is a natural derivative of the bioflavonoid rutin that is present in coffee, cereal grains, tea, and vegetables. The neuroprotective effects of troxerutin were reported in a 6-OHDA rat model to reduce striatal lipid peroxidation, ROS, GFAP, and DNA fragmentation. Meanwhile, troxerutin was capable of preventing loss of TH-positive neurons in the SN [54]. The bioflavonoid hesperidin is a specific flavonoid glycoside frequently found in oranges and lemons. Hesperidin protects against iron-induced oxidative damage and dopamine depletion in Drosophila melanogaster model of PD [55]. In addition, in 6-OHDA-treated mice, hesperidin protects by reducing oxidative damage, increasing the dopamine levels and also improving the behavioural parameters [56].

2.4. Valeriana officinalis. Valeriana officinalis (Valerian) is a plant with sedative and antispasmodic effect, traditionally used in the treatment of insomnia, anxiety, and restlessness. The effects of valerian on rotenone-induced cell death in $\mathrm{SH}$ SY5Y cells have been demonstrated [85]. Moreover, extract of valerian was effective in reducing the toxicity induced by rotenone in Drosophila melanogaster, as confirmed by the normalisation in the expression of SOD and catalase mRNAs, 
suggesting that the effects of valerian are, at least in part, associated with the antioxidant properties of the plant due to its phenolic and flavonoid constituents [57]. Valeriana wallichii, also known as Indian valerian or Tagar-Ganthoda, belongs to the family Valerianaceae and is considered as an important Asian counterpart of the European valerian. Thus, valeriana wallichii treatment significantly recuperated the altered behaviour, striatal dopamine levels, increased GFAP expression, and the histopathological changes observed in mice treated with MPTP. Likewise, it ameliorated the increased levels of ROS, inflammatory cytokines and lipid peroxidation and also ameliorated the diminished levels of antioxidants [58].

2.5. Passion Flower. Passion flower, commonly known as Passiflora incarnata (Passifloraceae), contains flavonoids, glycosides, alkaloids, and phenolic compounds. Also, it has been used for the treatment of anxiety, insomnia, epilepsy, muscular spasms, and other diseases [86]. Therefore, the biological effects of passion flower have been investigated in PD. The extract of passion flower reduced the number of jaw movements induced by tacrine, which is a widely used animal model of PD tremors. In addition, the model showed cognitive improvement, with significantly reduced duration of haloperidol-induced catalepsy. The passion flower possesses antioxidant activity, as shown by its significant scavenging ability [59]. Passiflora cincinnata is a Brazilian native species of passion flower and its possible biological effects have been investigated. Thus, in a model of PD induced by reserpine, Passiflora cincinnata extract prevented the decrease in $\mathrm{TH}$ in the SN induced by reserpine, delayed the onset of motor impairments, and prevented the occurrence of increased catalepsy behaviour. However, the extract did not modify reserpine-induced cognitive impairments [60].

2.6. St. John's Wort. The use of St. John's wort, known as Hypericum perforatum, dates back to the time of the ancient Greeks. Active compounds of St. John's wort have been identified and include naphthodianthrones, phloroglucinols, and flavonoids (such as phenylpropanes, flavonol glycosides, and biflavones), as well as essential oils. Therefore, the active compounds provide antioxidant and neuroprotective effects [87]. Two standardised extracts of St. John's wort have been tested on the neurodegeneration induced by chronic administration of rotenone in rats. Accordingly, St. John's wort reduced neuronal damage and inhibited the apoptotic cascade by decreasing Bax levels [61]. Besides, intrastriatal 6-OHDA-lesioned rats were treated with an the extract of St. John's wort and showed lowered striatal level of malondialdehyde, enhanced catalase activity, reduced GSH content, normalised expression of GFAP and $\mathrm{TNF} \alpha$, lowered DNA fragmentation and prevention of damage to dopaminergic neurons [62].

\section{Attention-Deficit/Hyperactivity Disorder}

Attention-deficit/hyperactivity disorder (ADHD) is the most common neurodevelopmental disorder in childhood and is characterised by inattention, impulsivity, and hyperactivity. The worldwide prevalence of ADHD in children and adolescents is $5.3 \%$ [88]; across cultures the prevalence is estimated to range from $5 \%$ to $7 \%[88,89]$. ADHD has high impact on school performance and causes impairments in personal, social, or occupational function, leading to isolation, poorer grades, and in adolescence there is increased risk of depression, delinquent and antisocial behaviour, incurring comorbid conditions, and later substance abuse [90]. ADHD has been associated with deregulation of the catecholaminergic pathway in the brain [91], although extensive data point to oxidative stress as potential contributor to the pathophysiology in ADHD [92]. Currently, ADHD treatment is pharmacologic and improves the attention, reducing distractibility and impulsive behaviour. Pharmacologic agents used for the treatment of ADHD increase levels of catecholamines in the brain, alleviating $\mathrm{ADHD}$ symptoms, and can be divided into two: psychostimulants and nonpsychostimulants [93]. Methylphenidate (MPH) is a psychostimulant that increases extracellular dopamine and norepinephrine levels, thereby correcting the underlying abnormalities in catecholaminergic functions and restoring neurotransmitter imbalance [94]. Atomoxetine (ATX) is a nonpsychostimulant, a norepinephrine specific reuptake inhibitor that increases catecholamine levels in the brain resulting in behavioural improvement $[94,95]$. ADHD is a heterogeneous disorder and is categorized into three subtypes: hyperactive/impulsive, inattentive, and the combined subtype $[89,90]$. There may be also differences in terms of treatment responses, for example, the hyperactive/impulsive subtype responds well to $\mathrm{MPH}$, the inattentive subtype responds better to ATX, and the combined type responds well to ATX and MPH, although controversy still persists about which is the best treatment for each subtype. However, it has been demonstrated that the pharmacological treatments to improve ADHD symptoms have some side effects, which include nausea, headache, insomnia, abdominal pain, decreased appetite, and motor tics [96]. Drugs used for the ADHD treatment and their side effects are shown in Table 1. Moreover, the use of psychostimulant medications in ADHD has been also associated with increased risk of substance use disorder [97]. Thus, there has been growing interest in alternative treatments for $\mathrm{ADHD}$, including natural compounds because of their antioxidant properties [98]. Natural compounds used as alternative therapies for the management of ADHD are shown in Table 3.

3.1. Ginkgo biloba. The extract from G. biloba leaves has been used as a herbal medicine for dementia [81, 99]. Therefore, several reports indicate that $G$. biloba may have therapeutic benefits in ADHD, given that the cotreatment with G. biloba and ginseng were found to alleviate ADHD symptoms in children, with minor side effects observed [63]. Moreover, in a double-blind, randomised trial the administration of $\mathrm{G}$. biloba was less effective than MPH in the treatment of ADHD [64]. G. biloba extract treatment at a maximal dosage of 240 mg improved the behavioural ratings of $\mathrm{ADHD}$ symptoms and the electrical brain activity in children, suggesting that G. biloba extract seems to be well tolerated in the short term 


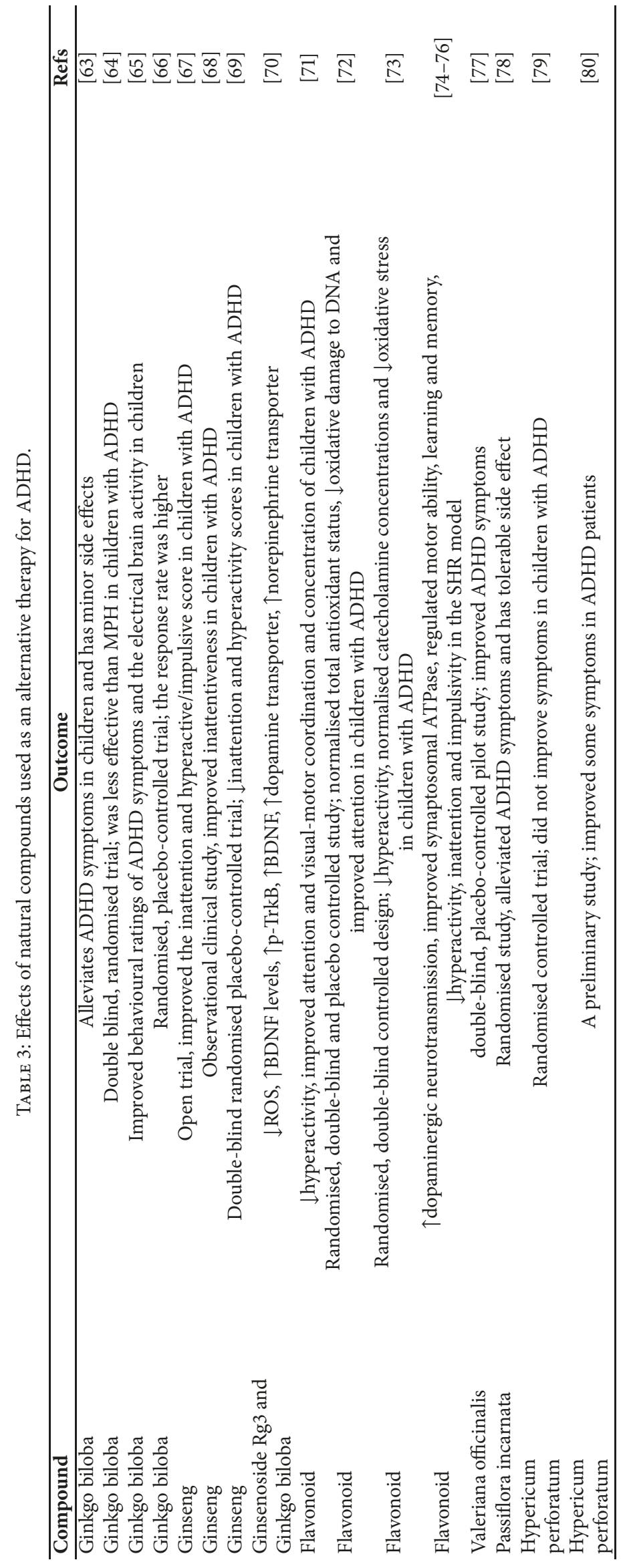


and may be a useful treatment [65]. Lately, in a randomised, placebo-controlled trial the response rate was higher with $\mathrm{G}$. biloba treatment compared to placebo. Thus, G. biloba could be an effective complementary treatment for ADHD [66].

3.2. Ginseng. Ginseng contains a class of phytochemicals called ginsenosides, which are known as potent antioxidants and for their neuroprotective properties. Ginseng has been shown to alleviate effectively symptoms of ADHD. In an open trial, ginseng medication improved the inattention and hyperactive/impulsive score in children with ADHD [67]. Indeed, an observational clinical study showed that ginseng, given at $1000 \mathrm{mg}$ for 8 weeks, improved inattentiveness in children with ADHD [68]. A double-blind randomised, placebo-controlled trial reported that ginseng decreased inattention and hyperactivity scores in children with ADHD [69]. Hence, ginseng has the potential to be used as an alternative therapy for ADHD. On the ADHD-like condition induced by Aroclor1254, YY162, which consists of terpenoidstrengthened G. biloba and ginsenoside $\mathrm{Rg} 3$, attenuated the increase in ROS and decrease in BDNF levels in SHSY5Y cells. Moreover, YY162 attenuated reductions in pTrkB, BDNF, dopamine transporter, and norepinephrine transporter expression [70].

3.3. Flavonoids. Pycnogenol is a herbal dietary supplement extracted from French maritime pine bark whose main ingredient is procyanidin. Procyanidins are members of the proanthocyanidin a class of flavonoids and are powerful antioxidants also found in food such as grapes, berries, pomegranates, red wine, and nuts. The treatment of 1-month pycnogenol administration resulted in a significant reduction in hyperactivity, improved attention, and visual-motor coordination and concentration in children with ADHD [71]. Moreover, pycnogenol reduced oxidative damage to DNA, normalised total antioxidant status, and improved attention, as demonstrated in a randomised, double-blind, placebocontrolled study [72]. Also, the administration of pycnogenol in children with ADHD, normalised catecholamine concentrations, leading to less hyperactivity, and reduced oxidative stress, in a randomised, double-blind, controlled design [73]. St. John's wort and pycnogenol have been tested as therapeutic alternatives to treat ADHD. A significant increase of $\mathrm{SH}$ SY5Y cell survival was induced by pycnogenol, which did not cause any cytotoxic effect when used in therapeutically relevant concentrations; also, treatment with St. John's wort significantly increased ATP levels [100]. Oroxylin A is a flavonoid isolated from the root of Scutellaria baicalensis Georgi, a herb found in East Asia. It has been observed that oroxylin A is an antagonist of the GABA-A receptor and its neuroprotective actions include antioxidant, antiinflammatory, and memory-enhancing effects. On the other hand, spontaneously hypertensive rats (SHRs) display some symptoms of $\mathrm{ADHD}$, which makes them a model of the disorder. It was demonstrated that oroxylin A improved ADHD-like behaviours via enhancement of dopaminergic neurotransmission and not the modulation of the GABA pathway in the SHR [74]. Furthermore, an oroxylin A analogue reduced hyperactivity, sustained inattention, and impulsivity in the SHR [75]. Baicalin regulated the motor ability and learning and memory abilities in the SHR and thus controlled the core symptoms of ADHD [76]. Also, baicalin improved synaptosomal ATPase and LDH activities in the SHR, suggesting that baicalin exerts its therapeutic effect by upregulating the AC/cAMP/PKA signalling pathway [101]. A randomised, double-blind trial to investigate the therapeutic benefit of pycnogenol in ADHD patients is in progress [102].

3.4. Valeriana officinalis. The efficacy of valerian has been evaluated in a double-blind, placebo-controlled pilot study, where valerian showed improvement in ADHD symptoms, in particular, sustained inattention, anxiety and impulsivity, and/or hyperactivity [77]. The GABA- A receptors are the substrate for the anxiolytic action of valerenic acid, a major constituent of valerian root extracts [103]. GABA is the main inhibitory neurotransmitter in the CNS, and its deficiency causes anxiety, restlessness, and obsessive behaviour, symptoms often seen in ADHD. The European Medicine Agency deemed that root extracts of valerian could be used for the relief of mild nervous tension and sleep disorders. However, more research is required to support the efficacy of valerian in the treatment $\mathrm{ADHD}$.

3.5. Passion Flower. The effect of passion flower in alleviating ADHD symptoms was tested in a randomised study. In addition, a tolerable side effect profile may be considered as one of the advantages of passion flower as compared with MPH [78]. It seems that the mechanism of action of passion flower is mediated via modulation of the GABA-A and GABA-B receptors and its effects on GABA uptake [104]. Although the passion flower has shown pharmacological activity in preclinical experiments, including sedative, anxiolytic, antitussive, antiasthmatic, and antidiabetic activities, its supposed efficacy does not appear to be adequately corroborated in the literature, since clinical studies often present methodologies and procedures with weaknesses [105].

3.6. St. John's Wort. St. John's wort produces its therapeutic effects by involving inhibition of the reuptake of dopamine, serotonin, and norepinephrine [106]. In a randomised controlled trial, the use of St. John's wort for the treatment of ADHD over the course of eight weeks did not improve symptoms [79]. However, a preliminary study reported that treatment with St. John's wort improved some symptoms in ADHD patients [80]. Because of findings that St. John's wort has no adverse effects, more studies are required to determine efficacy in the treatment of ADHD.

\section{Conclusions}

The natural compounds discussed in this review appear to be promising for the treatment of $\mathrm{PD}$ and ADHD. Therefore, these compounds can lay the foundation for a new therapeutic approach for the treatment of these disorders. Natural compounds are more easily accepted by patients, since they are considered healthier than synthetic drugs. Although the use of natural compounds for the neurological 
disorders has been considered as a safe approach, they are still far from being standard treatments, due to the lack of controlled clinical studies that could corroborate both their high efficacy and safety. Hence, better designed and more rigorous clinical trials are required before they can be established as therapeutic compounds. Neither PD nor ADHD, until today, have a therapeutic option capable of counteracting the progression of the disease, and natural compounds are often able to modulate the progression of this type of disorder, as demonstrated by their decreasing of oxidative stress.

\section{Conflicts of Interest}

The author declares that there are no conflicts of interest.

\section{Acknowledgments}

This work was supported by Fondos Federales HIM 2015/022 SSA 1160.

\section{References}

[1] W. Dauer and S. Przedborski, "Parkinson's disease: mechanisms and models," Neuron, vol. 39, no. 6, pp. 889-909, 2003.

[2] C. W. Olanow, D. R. Wakeman, and J. H. Kordower, "Peripheral alpha-synuclein and Parkinson's disease," Movement Disorders, vol. 29, no. 8, pp. 963-966, 2014.

[3] J. C. Corona and M. R. Duchen, "PPARgamma and PGC-1alpha as therapeutic targets in Parkinson's," Neurochemical Research, vol. 40, no. 2, pp. 308-316, 2015.

[4] H. Deng, P. Wang, and J. Jankovic, "The genetics of Parkinson disease," Ageing Research Reviews, vol. 42, pp. 72-85, 2018.

[5] L. Brichta, P. Greengard, and M. Flajolet, "Advances in the pharmacological treatment of Parkinson's disease: targeting neurotransmitter systems," Trends in Neurosciences, vol. 36, no. 9, pp. 543-554, 2013.

[6] B. K. Young, R. Camicioli, and L. Ganzini, "Neuropsychiatric adverse effects of antiparkinsonian drugs. Characteristics, evaluation and treatment," Drugs \& Aging, vol. 10, no. 5, pp. 367-383, 1997.

[7] H. Homayoun, "Parkinson Disease," Annals of Internal Medicine, vol. 169, no. 5, pp. ITC33-ITC48, 2018.

[8] R. D. White, G. D. Harris, and M. E. Gibson, "Attention Deficit Hyperactivity Disorder and Athletes," Sports Health, vol. 6, no. 2, pp. 149-156, 2014.

[9] C. Ramassamy, F. Clostre, Y. Christen, and J. Costentin, "Prevention by a Ginkgo biloba Extract (GBE 761) of the Dopaminergic Neurotoxicity of MPTP," Journal of Pharmacy and Pharmacology, vol. 42, no. 11, pp. 785-789, 1990.

[10] W.-R. Wu and X.-Z. Zhu, "Involvement of monoamine oxidase inhibition in neuroprotective and neurorestorative effects of Ginkgo biloba extract against MPTP-induced nigrostriatal dopaminergic toxicity in C57 mice," Life Sciences, vol. 65, no. 2, pp. 157-164, 1999.

[11] F. Cao, S. Sun, and E. T. Tong, "Experimental study on inhibition of neuronal toxical effect of levodopa by ginkgo biloba extract on Parkinson disease in rats," Journal of Huazhong University of Science and Technology - Medical Sciences, vol. 23, no. 2, pp. 151-153, 2003.
[12] M.-S. Kim, J.-I. Lee, W.-Y. Lee, and S.-E. Kim, "Neuroprotective effect of Ginkgo biloba L. extract in a rat model of parkinson's disease," Phytotherapy Research, vol. 18, no. 8, pp. 663-666, 2004.

[13] X. Kang, J. Chen, Z. Xu, H. Li, and B. Wang, "Protective effects of Ginkgo biloba extract on paraquat-induced apoptosis of PC12 cells," Toxicology in Vitro, vol. 21, no. 6, pp. 1003-1009, 2007.

[14] P. Rojas, N. Serrano-García, J. J. Mares-Sámano, O. N. MedinaCampos, J. Pedraza-Chaverri, and S. O. Ögren, "EGb761 protects against nigrostriatal dopaminergic neurotoxicity in 1methyl-4-phenyl-1,2,3, 6-tetrahydropyridine-induced Parkinsonism in mice: Role of oxidative stress," European Journal of Neuroscience, vol. 28, no. 1, pp. 41-50, 2008.

[15] M. A. El-Ghazaly, N. A. Sadik, E. R. Rashed, and A. A. AbdEl-Fattah, "Neuroprotective effect of EGb761(R) and low-dose whole-body gamma-irradiation in a rat model of Parkinson's disease," Toxicology \& Industrial Health, vol. 31, no. 12, pp. 11281143, 2015.

[16] Y. Q. Wang, M. Y. Wang, X. R. Fu et al., "Neuroprotective effects of ginkgetin against neuroinjury in Parkinson's disease model induced by MPTP via chelating iron," Free Radical Research, vol. 49, no. 9, pp. 1069-1080, 2015.

[17] J. Hua, N. Yin, B. Yang et al., "Ginkgolide B and bilobalide ameliorate neural cell apoptosis in $\alpha$-synuclein aggregates," Biomedicine \& Pharmacotherapy, vol. 96, pp. 792-797, 2017.

[18] S. Kuang, L. Yang, Z. Rao et al., "Effects of Ginkgo Biloba Extract on A53T $\alpha$-Synuclein Transgenic Mouse Models of Parkinson's Disease," Canadian Journal of Neurological Sciences, vol. 45, no. 2, pp. 182-187, 2018.

[19] M. Rudakewich, F. Ba, and C. G. Benishin, "Neurotrophic and neuroprotective actions of ginsenosides $\mathrm{Rb}(1)$ and $\mathrm{Rg}(1)$," Planta Medica, vol. 67, no. 6, pp. 533-537, 2001.

[20] X.-C. Chen, Y. Chen, Y.-G. Zhu, F. Fang, and L.-M. Chen, "Protective effect of ginsenoside Rg1 against MPTP-induced apoptosis in mouse substantia nigra neurons," Acta Pharmacologica Sinica, vol. 23, no. 9, pp. 829-834, 2002.

[21] J. Van Kampen, H. Robertson, T. Hagg, and R. Drobitch, "Neuroprotective actions of the ginseng extract G115 in two rodent models of Parkinson's disease," Experimental Neurology, vol. 184, no. 1, pp. 521-529, 2003.

[22] X.-C. Chen, Y.-C. Zhou, F. Fang, Y. Chen, Y.-G. Zhu, and L.M. Chen, "Ginsenoside Rg1 reduces MPTP-induced substantia nigra neuron loss by suppressing oxidative stress," Acta Pharmacologica Sinica, vol. 26, no. 1, pp. 56-62, 2005.

[23] S. Hu, R. Han, S. Mak, and Y. Han, "Protection against 1-methyl4-phenylpyridinium ion $\left(\mathrm{MPP}^{+}\right)$-induced apoptosis by water extract of ginseng (Panax ginseng C.A. Meyer) in SH-SY5Y cells," Journal of Ethnopharmacology, vol. 135, no. 1, pp. 34-42, 2011.

[24] W. Jiang, Z. Wang, Y. Jiang, M. Lu, and X. Li, "Ginsenoside Rg1 ameliorates motor function in an animal model of Parkinson's disease," Pharmacology, vol. 96, no. 1-2, pp. 25-31, 2015.

[25] M. T. Ardah, K. E. Paleologou, G. Lv et al., "Ginsenoside Rb1 inhibits fibrillation and toxicity of alpha-synuclein and disaggregates preformed fibrils," Neurobiology of Disease, vol. 74, pp. 89-101, 2015.

[26] T.-T. Zhou, G. Zu, X. Wang et al., "Immunomodulatory and neuroprotective effects of ginsenoside Rg1 in the $\operatorname{MPTP}(1-$ methyl-4-phenyl-1,2,3,6-tetrahydropyridine) -induced mouse model of Parkinson's disease," International Immunopharmacology, vol. 29, no. 2, pp. 334-343, 2015. 
[27] T. Zhou, G. Zu, X. Zhang et al., "Neuroprotective effects of ginsenoside Rg1 through the Wnt/ $\beta$-catenin signaling pathway in both in vivo and in vitro models of Parkinson's disease," Neuropharmacology, vol. 101, no. 5, pp. 480-489, 2016.

[28] Y. Heng, Q.-S. Zhang, Z. Mu, J.-F. Hu, Y.-H. Yuan, and N.H. Chen, "Ginsenoside Rg1 attenuates motor impairment and neuroinflammation in the MPTP-probenecid-induced parkinsonism mouse model by targeting $\alpha$-synuclein abnormalities in the substantia nigra," Toxicology Letters, vol. 243, pp. 7-21, 2016.

[29] E. González-Burgos, C. Fernández-Moriano, R. Lozano, I. Iglesias, and M. P. Gómez-Serranillos, "Ginsenosides Rd and Re co-treatments improve rotenone-induced oxidative stress and mitochondrial impairment in SH-SY5Y neuroblastoma cells," Food and Chemical Toxicology, vol. 109, pp. 38-47, 2017.

[30] Y. Liu, R.-Y. Zhang, J. Zhao et al., "Ginsenoside Rd protects SH-SY5Y cells against 1-methyl-4-phenylpyridinium induced injury," International Journal of Molecular Sciences, vol. 16, no. 7, pp. 14395-14408, 2015.

[31] H.-I. Im, W. S. Joo, E. Nam, E.-S. Lee, Y.-J. Hwang, and Y. S. Kim, "Baicalein prevents 6-hydroxydopamine-induced dopaminergic dysfunction and lipid peroxidation in mice," Journal of Pharmacological Sciences, vol. 98, no. 2, pp. 185-189, 2005.

[32] Y. Cheng, G. He, X. Mu et al., "Neuroprotective effect of baicalein against MPTP neurotoxicity: Behavioral, biochemical and immunohistochemical profile," Neuroscience Letters, vol. 441, no. 1, pp. 16-20, 2008.

[33] X. Mu, G. He, Y. Cheng, X. Li, B. Xu, and G. Du, "Baicalein exerts neuroprotective effects in 6-hydroxydopamine-induced experimental parkinsonism in vivo and in vitro," Pharmacology Biochemistry \& Behavior, vol. 92, no. 4, pp. 642-648, 2009.

[34] M. Jiang, Y. Porat-Shliom, Z. Pei et al., "Baicalein reduces E46K $\alpha$-synuclein aggregation in vitro and protects cells against E46K $\alpha$-synuclein toxicity in cell models of familiar Parkinsonism," Journal of Neurochemistry, vol. 114, no. 2, pp. 419-429, 2010.

[35] J.-H. Lu, M. T. Ardah, S. S. K. Durairajan et al., "Baicalein Inhibits Formation of $\alpha$-Synuclein Oligomers within Living Cells and Prevents $\mathrm{A} \beta$ Peptide Fibrillation and Oligomerisation," ChemBioChem, vol. 12, no. 4, pp. 615-624, 2011.

[36] Y.-H. Wang, H.-T. Yu, X.-P. Pu, and G.-H. Du, "Baicalein prevents 6-hydroxydopamine-induced mitochondrial dysfunction in SH-SY5Y cells via inhibition of mitochondrial oxidation and up-regulation of DJ-1 protein expression," Molecules, vol. 18, no. 12, pp. 14726-14738, 2013.

[37] E. Lee, H. R. Park, S. T. Ji, Y. Lee, and J. Lee, “Baicalein attenuates astroglial activation in the 1-methyl-4-phenyl-1,2,3,4-tetrahydropyridine-induced Parkinson's disease model by downregulating the activations of nuclear factor- $\kappa \mathrm{B}$, ERK, and JNK," Journal of Neuroscience Research, vol. 92, no. 1, pp. 130-139, 2014.

[38] X. Xue, H. Liu, L. Qi et al., "Baicalein ameliorated the upregulation of striatal glutamatergic transmission in the mice model of Parkinson's disease," Brain Research Bulletin, vol. 103, pp. 54-59, 2014.

[39] Q. Hu, V. N. Uversky, M. Huang et al., "Baicalein inhibits alpha-synuclein oligomer formation and prevents progression of alpha-synuclein accumulation in a rotenone mouse model of Parkinson's disease," Biochim Biophys Acta, vol. 1862, no. 10, pp. 1883-1890, 2016.

[40] K.-C. Hung, H.-J. Huang, Y.-T. Wang, and A. M.-Y. Lin, "Baicalein attenuates $\alpha$-synuclein aggregation, inflammasome activation and autophagy in the MPP+-treated nigrostriatal dopaminergic system in vivo," Journal of Ethnopharmacology, vol. 194, pp. 522-529, 2016.
[41] H.-Q. Chen, Z.-Y. Jin, X.-J. Wang, X.-M. Xu, L. Deng, and J.-W. Zhao, "Luteolin protects dopaminergic neurons from inflammation-induced injury through inhibition of microglial activation," Neuroscience Letters, vol. 448, no. 2, pp. 175-179, 2008.

[42] L. Hu, J. Yen, Y. Shen, K. Wu, M. Wu, and Y. Ho, "Luteolin Modulates 6-Hydroxydopamine-Induced Transcriptional Changes of Stress Response Pathways in PC12 Cells," PLoS ONE, vol. 9, no. 5, p. e97880, 2014.

[43] S. P. Patil, P. D. Jain, J. S. Sancheti, P. J. Ghumatkar, R. Tambe, and S. Sathaye, "Neuroprotective and neurotrophic effects of Apigenin and Luteolin in MPTP induced parkinsonism in mice," Neuropharmacology, vol. 86, pp. 192-202, 2014.

[44] N. Haleagrahara, C. J. Siew, N. K. Mitra, and M. Kumari, "Neuroprotective effect of bioflavonoid quercetin in 6hydroxydopamine-induced oxidative stress biomarkers in the rat striatum," Neuroscience Letters, vol. 500, no. 2, pp. 139-143, 2011.

[45] C. Lv, T. Hong, Z. Yang et al., "Effect of quercetin in the 1methyl-4-phenyl-1, 2, 3, 6-tetrahydropyridine-induced mouse model of parkinson's disease," Evidence-Based Complementary and Alternative Medicine, vol. 2012, Article ID 928643, 6 pages, 2012.

[46] S. S. Karuppagounder, S. K. Madathil, M. Pandey, R. Haobam, U. Rajamma, and K. P. Mohanakumar, "Quercetin up-regulates mitochondrial complex-I activity to protect against programmed cell death in rotenone model of Parkinson's disease in rats," Neuroscience, vol. 236, pp. 136-148, 2013.

[47] M. Ay, J. Luo, M. Langley et al., "Molecular mechanisms underlying protective effects of quercetin against mitochondrial dysfunction and progressive dopaminergic neurodegeneration in cell culture and MitoPark transgenic mouse models of Parkinson's Disease," Journal of Neurochemistry, vol. 141, no. 5, pp. 766-782, 2017.

[48] S. Li and X.-P. Pu, "Neuroprotective effect of kaempferol against a 1-methyl-4-phenyl-1,2,3,6-tetrahydropyridine-induced mouse model of Parkinson's disease," Biological \& Pharmaceutical Bulletin, vol. 34, no. 8, pp. 1291-1296, 2011.

[49] G. Filomeni, I. Graziani, D. de Zio et al., "Neuroprotection of kaempferol by autophagy in models of rotenone-mediated acute toxicity: possible implications for Parkinson's disease," Neurobiology of Aging, vol. 33, no. 4, pp. 767-785, 2012.

[50] M. M. Khan, S. S. Raza, H. Javed et al., "Rutin protects dopaminergic neurons from oxidative stress in an animal model of Parkinson's disease," Neurotoxicity Research, vol. 22, no. 1, pp. $1-15,2012$.

[51] K. B. Magalingam, A. Radhakrishnan, and N. Haleagrahara, "Rutin, a bioflavonoid antioxidant protects rat pheochromocytoma (PC-12) cells against 6-hydroxydopamine (6-OHDA)induced neurotoxicity," International Journal of Molecular Medicine, vol. 32, no. 1, pp. 235-240, 2013.

[52] K. B. Magalingam, A. Radhakrishnan, and N. Haleagrahara, "Protective effects of flavonol isoquercitrin, against 6hydroxydopamine (6-OHDA)-induced toxicity in PC12 cells," BMC Research Notes, vol. 7, p. 49, 2014.

[53] C. Anusha, T. Sumathi, and L. D. Joseph, "Protective role of apigenin on rotenone induced rat model of Parkinson's disease: Suppression of neuroinflammation and oxidative stress mediated apoptosis," Chemico-Biological Interactions, vol. 269, pp. 67-79, 2017.

[54] T. Baluchnejadmojarad, N. Jamali-Raeufy, S. Zabihnejad, N. Rabiee, and M. Roghani, "Troxerutin exerts neuroprotection 
in 6-hydroxydopamine lesion rat model of Parkinson's disease: Possible involvement of PI3K/ER $\beta$ signaling," European Journal of Pharmacology, vol. 801, pp. 72-78, 2017.

[55] M. R. Poetini, S. M. Araujo, M. Trindade de Paula et al., "Hesperidin attenuates iron-induced oxidative damage and dopamine depletion in Drosophila melanogaster model of Parkinson's disease," Chemico-Biological Interactions, vol. 279, pp. 177-186, 2018.

[56] M. S. Antunes, A. T. R. Goes, S. P. Boeira, M. Prigol, and C. R. Jesse, "Protective effect of hesperidin in a model of Parkinson's disease induced by 6-hydroxydopamine in aged mice," Nutrition Journal, vol. 30, no. 11-12, pp. 1415-1422, 2014.

[57] J. H. Sudati, F. A. Vieira, and S. S. Pavin, "Valeriana officinalis attenuates the rotenone-induced toxicity in Drosophila melanogaster," NeuroToxicology, vol. 37, pp. 118-126, 2013.

[58] S. Sridharan, K. Mohankumar, S. P. Jeepipalli et al., "Neuroprotective effect of Valeriana wallichii rhizome extract against the neurotoxin MPTP in C57BL/6 mice," NeuroToxicology, vol. 51, pp. 172-183, 2015.

[59] S. Ingale and S. Kasture, "Protective effect of standardized extract of Passiflora incarnata flower in parkinson's and alzheimer's disease," Ancient Science of Life, vol. 36, no. 4, pp. 200-206, 2017.

[60] L. E. M. Brandão and D. Nôga, "Passiflora cincinnata extract delays the development of motor signs and prevents dopaminergic loss in a mice model of parkinson's disease," EvidenceBased Complementary and Alternative Medicine, vol. 2017, Article ID 8429290, 11 pages, 2017.

[61] M. A. Gómez del Rio, M. I. Sánchez-Reus, I. Iglesias et al., "Neuroprotective properties of standardized extracts of hypericum perforatum on rotenone model of parkinson's disease," CNS and Neurological Disorders - Drug Targets, vol. 12, no. 5, pp. 665-679, 2013.

[62] Z. Kiasalari, T. Baluchnejadmojarad, and M. Roghani, "Hypericum perforatum hydroalcoholic extract mitigates motor dysfunction and is neuroprotective in intrastriatal 6-hydroxydopamine rat model of parkinson's disease," Cellular and Molecular Neurobiology, vol. 36, no. 4, pp. 521-530, 2016.

[63] M. R. Lyon, J. C. Cline, J. T. De Zepetnek, J. J. Shan, P. Pang, and C. Benishin, "Effect of the herbal extract combination Panax quinquefolium and Ginkgo biloba on attention-deficit hyperactivity disorder: a pilot study," Journal of Psychiatry \& Neuroscience, vol. 26, no. 3, pp. 221-228, 2001.

[64] B. Salehi, R. Imani, M. R. Mohammadi et al., "Ginkgo biloba for attention-deficit/hyperactivity disorder in children and adolescents: a double blind, randomized controlled trial," Progress in Neuro-Psychopharmacology \& Biological Psychiatry, vol. 34, no. 1, pp. 76-80, 2010.

[65] H. Uebel-von Sandersleben, A. Rothenberger, B. Albrecht, L. G. Rothenberger, S. Klement, and N. Bock, "Ginkgo biloba extract EGb 761 in children with ADHD," Zeitschrift für Kinder- und Jugendpsychiatrie und Psychotherapie, vol. 42, no. 5, pp. 337-347, 2014.

[66] F. Shakibaei, M. Radmanesh, E. Salari, and B. Mahaki, "Ginkgo biloba in the treatment of attention-deficit/hyperactivity disorder in children and adolescents. A randomized, placebocontrolled, trial," Complementary Therapies in Clinical Practice, vol. 21, no. 2, pp. 61-67, 2015.

[67] H. Niederhofer, "Panax ginseng may improve some symptoms of attention-deficit hyperactivity disorder," Journal of Dietary Supplements, vol. 6, no. 1, pp. 22-27, 2009.
[68] S. H. Lee, W. S. Park, and M. H. Lim, "Clinical effects of korean red ginseng on attention deficit hyperactivity disorder in children: an observational study," Journal of Ginseng Research, vol. 35, no. 2, pp. 226-234, 2011.

[69] H.-J. Ko, I. Kim, J.-B. Kim et al., "Effects of Korean red ginseng extract on behavior in children with symptoms of inattention and hyperactivity/impulsivity: a double-blind randomized placebo-controlled trial," Journal of Child and Adolescent Psychopharmacology, vol. 24, no. 9, pp. 501-508, 2014.

[70] Y. Nam, E.-J. Shin, S. W. Shin et al., "YY162 prevents ADHDlike behavioral side effects and cytotoxicity induced by Aroclor1254 via interactive signaling between antioxidant potential, BDNF/TrkB, DAT and NET," Food and Chemical Toxicology, vol. 65, pp. 280-292, 2014.

[71] J. Trebatická, S. Kopasová, Z. Hradečná et al., “Treatment of ADHD with French maritime pine bark extract, Pycnogenol," European Child and Adolescent Psychiatry, vol. 15, no. 6, pp. 329335, 2006.

[72] Z. Chovanová, J. Muchová, M. Sivoňová et al., "Effect of polyphenolic extract, Pycnogenol, on the level of 8-oxoguanine in children suffering from attention deficit/hyperactivity disorder," Free Radical Research, vol. 40, no. 9, pp. 1003-1010, 2006.

[73] M. Dvořáková, M. Sivoňová, J. Trebatická et al., “The effect of polyphenolic extract from pine bark, Pycnogenol, on the level of glutathione in children suffering from attention deficit hyperactivity disorder (ADHD)," Redox Report, vol. 11, no. 4, pp. 163-172, 2006.

[74] S. Y. Yoon, I. D. Peña, S. M. Kim et al., "Oroxylin A improves attention deficit hyperactivity disorder-like behaviors in the spontaneously hypertensive rat and inhibits reuptake of dopamine in vitro," Archives of Pharmacal Research, vol. 36, no. 1, pp. 134-140, 2013.

[75] I. C. Dela Peña, S. Y. Yoon, Y. Kim et al., "5,7-Dihydroxy6-methoxy- $4^{\prime}$-phenoxyflavone, a derivative of oroxylin A improves attention-deficit/hyperactivity disorder (ADHD)-like behaviors in spontaneously hypertensive rats," European Journal of Pharmacology, vol. 715, no. 1-3, pp. 337-344, 2013.

[76] R. Zhou, X. Han, J. Wang, and J. Sun, "Effect of baicalin on behavioral characteristics of rats with attention deficit hyperactivity disorder," Zhongguo Dang Dai Er Ke Za Zhi, vol. 19, no. 8, pp. 930-937, 2017.

[77] R. Razlog, J. Pellow, and S. J. White, "A pilot study on the efficacy of Valeriana officinalis mother tincture and Valeriana officinalis $3 \mathrm{X}$ in the treatment of attention deficit hyperactivity disorder," Health SA Gesondheid, vol. 17, no. 1, pp. 1-7, 2012.

[78] S. Akhondzadeh, M. R. Mohammadi, and F. Momeni, "Passiflora incarnata in the treatment of attention-deficit hyperactivity disorder in children and adolescents," Therapy, vol. 2, no. 4, pp. 609-614, 2005.

[79] W. Weber, A. Vander Stoep, R. L. McCarty, N. S. Weiss, J. Biederman, and J. McClellan, "Hypericum perforatum (St John's Wort) for attention-deficit/hyperactivity disorder in children and adolescents: a randomized controlled trial," The Journal of the American Medical Association, vol. 299, no. 22, pp. 26332641, 2008.

[80] H. Niederhofer, “St. John's wort may improve some symptoms of attention-deficit hyperactivity disorder," Natural Product Research, vol. 24, no. 3, pp. 203-205, 2010.

[81] F. V. DeFeudis and K. Drieu, "Ginkgo biloba extract (EGb 761) and CNS functions: basic studies and clinical applications," Current Drug Targets, vol. 1, no. 1, pp. 25-58, 2000. 
[82] P. Rojas, P. Montes, C. Rojas, N. Serrano-García, and J. C. RojasCastañeda, "Effect of a phytopharmaceutical medicine, Ginko biloba extract 761, in an animal model of Parkinson's disease: Therapeutic perspectives," Nutrition Journal , vol. 28, no. 11-12, pp. 1081-1088, 2012.

[83] C. Rendeiro, J. S. Rhodes, and J. P. E. Spencer, “The mechanisms of action of flavonoids in the brain: Direct versus indirect effects," Neurochemistry International, vol. 89, pp. 126-139, 2015.

[84] H. D. Kim, K. H. Jeong, U. J. Jung, and S. R. Kim, "Naringin treatment induces neuroprotective effects in a mouse model of Parkinson's disease in vivo, but not enough to restore the lesioned dopaminergic system," The Journal of Nutritional Biochemistry, vol. 28, pp. 140-146, 2016.

[85] D. M. de Oliveria, G. Barreto, D. V. G. de Andrade et al., "Cytoprotective effect of valeriana officinalis extract on an in vitro experimental model of parkinson disease," Neurochemical Research, vol. 34, no. 2, pp. 215-220, 2009.

[86] K. Dhawan, S. Dhawan, and A. Sharma, "Passiflora: a review update," Journal of Ethnopharmacology, vol. 94, no. 1, pp. 1-23, 2004.

[87] J. Barnes, L. A. Anderson, and J. D. Phillipson, "St John's wort (Hypericum perforatum L.): a review of its chemistry, pharmacology and clinical properties," Journal of Pharmacy and Pharmacology, vol. 53, no. 5, pp. 583-600, 2001.

[88] G. Polanczyk, M. S. de Lima, B. L. Horta, J. Biederman, and L. A. Rohde, "The worldwide prevalence of ADHD: a systematic review and metaregression analysis," The American Journal of Psychiatry, vol. 164, no. 6, pp. 942-948, 2007.

[89] E. G. Willcutt, "The prevalence of DSM-IV attention-deficit/ hyperactivity disorder: a meta-analytic review," Neurotherapeutics, vol. 9, no. 3, pp. 490-499, 2012.

[90] S. V. Faraone, P. Asherson, T. Banaschewski et al., "Attentiondeficit/hyperactivity disorder," Nature Reviews Disease Primers, vol. 1, p. 15020, 2015.

[91] J. Prince, "Catecholamine dysfunction in attention-deficit/ hyperactivity disorder: an update," Journal of Clinical Psychopharmacology, vol. 28, no. 3, suppl 2, pp. S39-S45, 2008.

[92] A. L. Lopresti, "Oxidative and nitrosative stress in ADHD: possible causes and the potential of antioxidant-targeted therapies," ADHD Attention Deficit and Hyperactivity Disorders, vol. 7, no. 4, pp. 237-247, 2015.

[93] L. Briars and T. Todd, "A review of pharmacological management of attention-deficit/hyperactivity disorder," The Journal of Pediatric Pharmacology and Therapeutics, vol. 21, no. 3, pp. 192206, 2016.

[94] J. Biederman, T. Spencer, and T. Wilens, "Evidence-based pharmacotherapy for attention-deficit hyperactivity disorder," The International Journal of Neuropsychopharmacology, vol. 7, no. 1, pp. 77-97, 2004.

[95] V. A. Reed, J. K. Buitelaar, E. Anand et al., "The safety of atomoxetine for the treatment of children and adolescents with attention-deficit/hyperactivity disorder: a comprehensive review of over a decade of research," CNS Drugs, vol. 30, no. 7, pp. 603-628, 2016.

[96] J. Lee, N. Grizenko, V. Bhat, S. Sengupta, A. Polotskaia, and R. Joober, "Relation between therapeutic response and side effects induced by methylphenidate as observed by parents and teachers of children with ADHD," BMC Psychiatry, vol. 11, p. 70, 2011.

[97] T. E. Wilens, A. Kwon, S. Tanguay et al., "Characteristics of adults with attention deficit hyperactivity disorder plus substance use disorder: The role of psychiatric comorbidity," American Journal on Addictions, vol. 14, no. 4, pp. 319-327, 2005.

[98] H. R. Searight, K. Robertson, T. Smith, S. Perkins, and B. K. Searight, "Complementary and Alternative Therapies for Pediatric Attention Deficit Hyperactivity Disorder: A Descriptive Review," ISRN Psychiatry, vol. 2012, Article ID 804127, 8 pages, 2012.

[99] P. L. Le Bars, M. M. Katz, N. Berman, T. M. Itil, A. M. Freedman, and A. F. Schatzberg, "A placebo-controlled, double-blind, randomized trial of an extract of Ginkgo biloba for dementia. North American EGb Study Group," The Journal of the American Medical Association, vol. 278, no. 16, pp. 1327-1332, 1997.

[100] A. J. Schmidt, J.-C. Krieg, U. M. Hemmeter et al., "Impact of plant extracts tested in attention-deficit/hyperactivity disorder treatment on cell survival and energy metabolism in human neuroblastoma SH-SY5Y cells," Phytotherapy Research, vol. 24, no. 10, pp. 1549-1553, 2010.

[101] R.-Y. Zhou, J.-J. Wang, Y. You et al., "Effect of baicalin on ATPase and $\mathrm{LDH}$ and its regulatory effect on the AC/cAMP/PKA signaling pathway in rats with attention deficit hyperactivity disorder," Zhongguo Dang Dai Er Ke Za Zhi, vol. 19, no. 5, pp. 576-582, 2017.

[102] A. A. J. Verlaet, B. Ceulemans, H. Verhelst et al., "Effect of Pycnogenol(R) on attention-deficit hyperactivity disorder (ADHD): study protocol for a randomised controlled trial," Trials, vol. 18, no. 1, p. 145, 2017.

[103] D. Benke, A. Barberis, S. Kopp et al., "GABAA receptors as in vivo substrate for the anxiolytic action of valerenic acid, a major constituent of valerian root extracts," Neuropharmacology, vol. 56, no. 1, pp. 174-181, 2009.

[104] K. Appel, T. Rose, B. Fiebich, T. Kammler, C. Hoffmann, and G. Weiss, "Modulation of the $\gamma$-aminobutyric acid (GABA) system by Passiflora incarnata L.," Phytotherapy Research, vol. 25, no. 6, pp. 838-843, 2011.

[105] M. Miroddi, G. Calapai, M. Navarra, P. L. Minciullo, and S. Gangemi, "Passiflora incarnata L.: ethnopharmacology, clinical application, safety and evaluation of clinical trials," Journal of Ethnopharmacology, vol. 150, no. 3, pp. 791-804, 2013.

[106] V. Butterweck, "Mechanism of action of St John's wort in depression: what is known?" CNS Drugs, vol. 17, no. 8, pp. 539$562,2003$. 

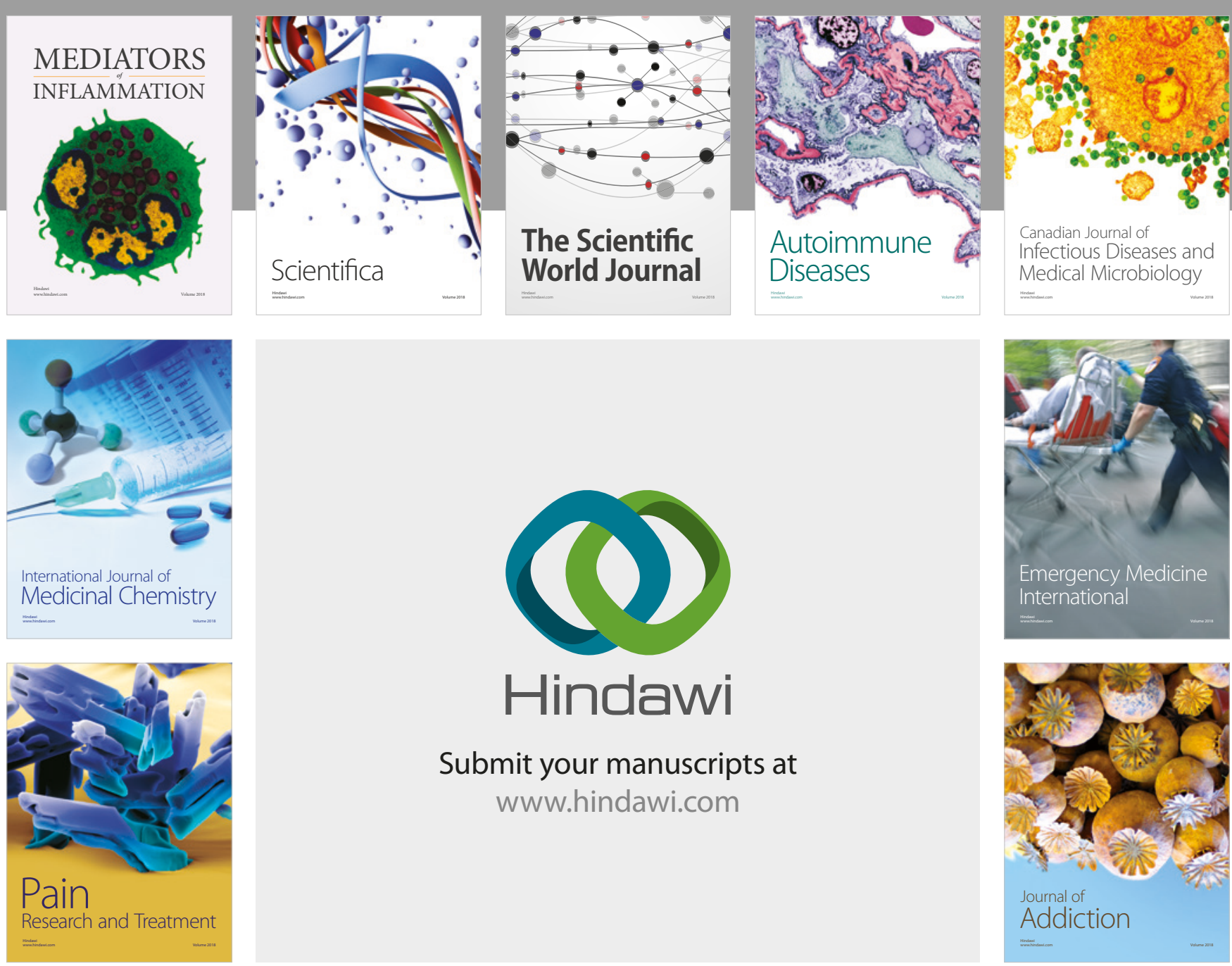

Canadian Journal of
Infectious Diseases and Medical Microbiology

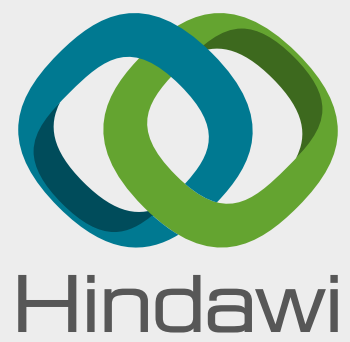

Submit your manuscripts at

www.hindawi.com
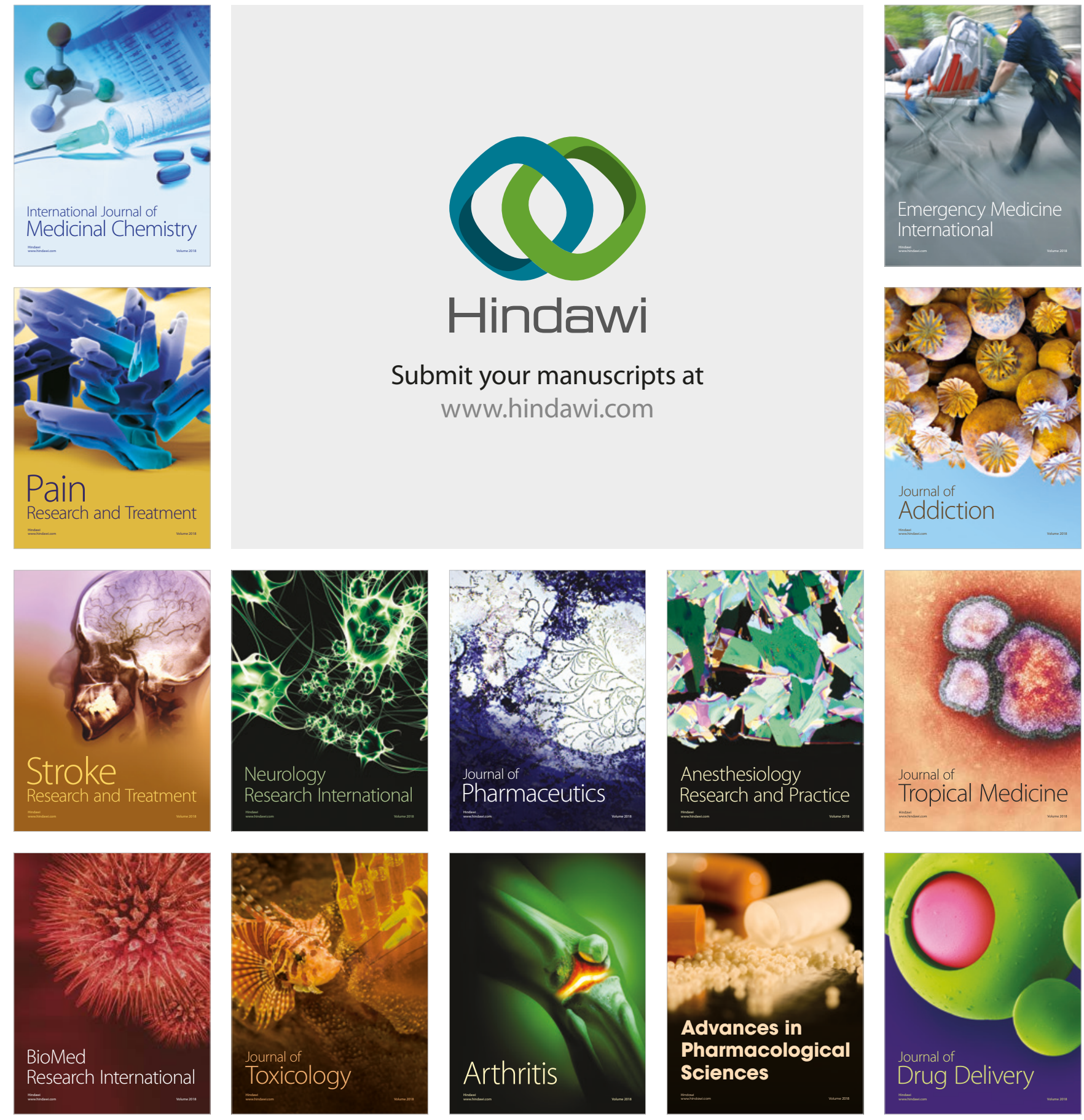